Sharif University of Technology
Scientia Iranica
Transactions E: Industrial Engineering
wCIENTIA

\title{
A robust optimization approach for an inventory problem with emergency ordering and product substitution in an uncertain environment: A case study in pharmaceutical industry
}

\author{
E. Mardan*, M. Sadegh Amalnick, M. Rabbani and F. Jolai \\ Department of Industrial Engineering, College of Engineering, University of Tehran, Tehran, Iran. \\ Received 23 February 2015; received in revised form 3 April 2016; accepted 24 May 2016
}

\author{
KEYWORDS \\ Substitutable \\ products; \\ Emergency ordering; \\ Inventory problem; \\ Yield uncertainty; \\ Inventory problem.
}

\begin{abstract}
This paper presents a multi-product, multi-period inventory problem in an uncertain environment where the main suppliers are prone to yield uncertainty. In order to overcome the arisen uncertainties, two basic approaches of emergency ordering and product substitutability are taken into consideration. In the proposed emergency ordering scheme, two sets of suppliers, i.e. cheap unreliable and expensive reliable (emergency) suppliers, are considered and a tradeoff between the cheap price of the main suppliers and reliability of emergency supplier is attained. In product substitution scheme, the demand of each product is fulfilled directly by the related product or other substitute products. A riskaverse decision maker is taken into consideration whose risk-averseness level is controlled by the portion of demand which should be definitely satisfied and not backordered or lost. A robust optimization approach with two variability measures is proposed to minimize the variability of the model. The results reveal the value of emergency ordering and product substitution. In addition, the results suggest which measure should be selected according to the decision maker's attitude toward the desired profit, variability, and service level.

(C) 2017 Sharif University of Technology. All rights reserved.
\end{abstract}

\section{Introduction}

In the last few decades, by the expansion of global business networks, organizations have tended to concentrate only on their core competitive capabilities and preferred to outsource the other portion of their activities. Although such practices can improve financial and operational performance of organizations in a problem-free environment, they can leave inverse consequences due to the high dependencies at time

*. Corresponding author. Tel.: +982188021067;

Fax: +982188013102

E-mail addresses: ehsanmardan@ut.ac.ir (E. Mardan); amalnick@ut.ac.ir (M. Sadegh Amalnik); mrabani@ut.ac.ir (M.Rabbani); fjolai@ut.ac.ir (F. Jolai) of disruption. Having no contingency, i.e. plan B, to protect the system against uncertainties, organizations will suffer the associated unexpected losses. This paper presents a multi-period, multi-product inventory problem with uncertainty in the supply of products and two types of flexibilities, i.e. emergency ordering and product substitution, are proposed to manage the effect of the arisen uncertainties. In emergency ordering scheme, it is possible to place orders on both cheap, unreliable suppliers prone to yield uncertainty and expensive reliable (emergency) supplier. Although unreliable suppliers have lower prices, the emergency supplier may outperform the cheap suppliers due to its reliability. Accordingly, in a proper ordering policy, a tradeoff between reliability and profitability of suppliers is achieved. In several contemporary examples, 
emergency ordering option has proved its effectiveness by the additional flexibilities provided for the decision maker. For instance, after a fire at Aisin Seiki Co., the main $P$-valve supplier of Toyota, which decreased its production capacity, Toyota called several flexible part-makers to manufacture the required $P$-valves. Somic was able to supply the $P$-valve configuration of Toyota by freeing up machines and delivered the required $P$-valves right on schedule. Taking advantage of emergency order to Somic, Toyota was able to leap a huge financial loss [1]. Besides emergency ordering decision, this paper jointly deals with product substitutability as another tool to handle the arisen uncertainties. Product substitution is applicable in a variety of industries including pharmaceutical, semiconductor, chemical industries, etc. For instance, in pharmaceutical industries, it is possible to use products with similar clinical effects interchangeably. In addition, in semiconductor industries, a high-performance product can be substituted with a similar yet lowperformance product. In several contemporary examples, product substitutability has emerged as a valuable option for managers. For instance, after a fire ignited by lightening at Royal Philips, the main supplier of Nokia microchips, Nokia substituted other compatible microchips, supplied from US and Japan, in its products to prevent market loss and customer dissatisfaction [2].

This paper presents an integrated model of product substitutability and emergency ordering. Although implementation of the emergency ordering or product substitutability concepts can improve the quality of solutions per se, considering an integrated model with emergency ordering and product substitutability can synergistically improve the quality of solutions. This arises due to the fact that emergency ordering option will improve the quality of procurement part while product substitutability option improves selling part of the model. Accordingly, considering an integrated model of emergency ordering and product substitutability can significantly improve the quality of the overall solutions and lead to higher net profit level. Clearly, the outcome of the model basically depends on the outcome of uncertain parameters. In this paper, to deal with uncertainties in the optimization models, a two-stage stochastic programing model and its robust extension are applied.

The main contribution of this paper can be summarized as follows: incorporation of emergency ordering and product substitutability into a nondeterministic inventory problem, in which unreliable suppliers are prone to yield uncertainty; and implementation of a robust approach as a novel tool considering the current limitations on the solution methodologies of inventory problems with uncertainties.

This paper is organized as follows. In Section 2, the related literature is presented with respect to the different related features of the problem. In Section 3, the mathematical model is presented. In Section 4, the numerical analysis of the case study is presented and the paper is concluded in Section 5 .

\section{Literature review}

By the outbreak of disruption and, accordingly, probable uncertainties in practical supply chains, numerous studies have been performed on business continuity frameworks, which desire to diminish the effects of disruptions on supply chains, e.g. [3,4]. Researchers have studied the effect of disruption on supply chains by different approaches. This paper studies an inventory problem with yield uncertainty in which the decision maker desires to improve the performance of the supply chain by the flexibilities prepared by emergency ordering and product substitution. Accordingly, our work is closely related to two streams of research in the literature including emergency ordering and product substitutability in inventory models with uncertainty.

In many practical implementations, the delivered quantity does not match the ordered quantities. Such concept can be modeled by uncertainty in the yield of suppliers. Qi and Shen [5] considered an EOQ model in which the suppliers were prone to yield uncertainty. Mukhopadhyay and Mu [6] studied a single-period model, which evaluated the optimal procurement quantities from unreliable suppliers prone to yield uncertainty. Agrawal and Nahmias [7] studied supplier diversification with deterministic demand and random yield of the supplier for a newsvendor inventory problem while Dada et al. [8] considered similar assumptions with stochastic demand in which the goal was to select a number of suppliers from the available set of suppliers. Maddah et al. [9] studied a production/inventory system with random yield in quality of products. Having a high capability to adopt supply uncertainty, random yield models are largely utilized in inventory models with uncertainties.

Only a small portion of the literature focuses on multiple sourcing strategies. Several studies have been performed in a single sourcing environment [10-12]. Not considering alternative suppliers, the above papers tend to keep extra inventory to decrease the effect of uncertainties on the supplier. Dada et al. [8] studied a newsvendor problem in which multiple unreliable suppliers with different prices and reliabilities were utilized to serve the customer demand. In most of the models with multiple suppliers, the prices of suppliers are considered to be similar while a portion of multiple sourcing models consider contingency sourcing tactic in which the orders are placed on both cheap unreliable and expensive reliable suppliers. Tomlin and Wang [13] contributed a dual sourcing model with two sets of 
cheap unreliable and expensive reliable suppliers. In the related research, the reliable supplier is also called the emergency supplier. Similarly, Tomlin [14] considered two sets of reliable and unreliable suppliers with capacity constraint and flexibility in the capacity of reliable supplier. Iakovou et al. [15] proposed a model in which the optimal reserved capacity level from the emergency supplier was determined for each discrete part by taking into account all inventory-related costs, including the premium cost to be paid for emergency capacity reservation. Keramydas et al. [16] focused on the evaluation of emergency sourcing risk mitigation strategies for a discrete part manufacturer, employing a quantitative approach. Chen and Yang [17] considered a supply chain in which a buyer purchased finished items from a contracting supplier to satisfy a stochastic market demand, where the supplier's production was subject to random yield. Mardan et al. [18] provided a two-stage stochastic model to handle the yield uncertainty of suppliers. Zhang et al. [19] provided comprehensive contingency management framework for supply chain disruption risk. Glud Johansen and Thorstenson [20] investigated emergency ordering in a periodic-review inventory system with fixed ordering cost and stochastic demand. Huang et al. [21] studied a real-time decision rule for inventory system with committed service time and emergency ordering. Axsäter [22] presented an improved decision rule for emergency replenishment policy. In the above models, emergency ordering has considerably improved the effectiveness of the associated models.

In the mentioned models, it is considered that the demand of each product can be fulfilled only by the related product while, in many practical applications, it is possible to satisfy the arisen demand exactly by the related product or substitutable products. Bassok et al. [23] studied a single-period model with periodic review with product substitutability. Ganesh et al. [24] studied the effect of product substitution in a multi-product, multi-level supply chain. Iravani et al. [25] studied process flexibility and inventory flexibility, which arose due to product substitutability. Huang et al. [26] studied a newsvendor problem with partial product substitution and proposed an iterative algorithm. Liu et al. [27] studied a newsvendor game with product substitution for two retailers. Goyal and Netessine [28] analyzed the volume flexibility for two products with substitution of products. This paper tends to present the mutual effect of product substitutability and emergency ordering as two novel tools to handle the arisen uncertainties.

In conclusion, it can be mentioned that there are a variety of methodologies for inventory models with suppliers yield uncertainty [29]. However, to the best of our knowledge, robust optimization modeling has not been implemented in the investigated area. In addition, product substitutability and emergency ordering have not been concurrently taken into consideration while considering both of these concepts can highly improve both procurement and demand fulfillment parts of the model. Accordingly, this paper has been established on two major gaps in the literature. First, this study considers the effect of both emergency ordering and product substitutability as efficient tools in minimizing the effect of supply uncertainties. Second, this paper considers a robust optimization approach, which can efficiently minimize the variability of the possible outcomes.

\section{Proposed model}

\subsection{Model assumptions}

This paper presents a multi-period inventory problem with multiple products/items. The items can be ordered into cheap unreliable or expensive reliable suppliers. The unreliability of suppliers arises due to the yield uncertainty. The demand can be fulfilled exactly by the related product or by the substitute product. The model is embedded in a two-stage decision making process in which, in the first stage, the orders are released to the unreliable suppliers and, later, when new information about the yield of the unreliable suppliers becomes available, the orders are released to the emergency reliable supplier. In addition, the assignment of the delivered products, which includes substitution of products, is decided in the second stage. It should be noted that the emergency orders, which are placed in the second stage, cannot exceed the reserved quantity of each item, which is determined in the first stage. Such assumption arises by the fact that the occasional cooperation with emergency suppliers can be performed based on reservation contracts. In addition, the surplus quantity of each item at the end of the planning horizon is considered in the model by a book value less than the procurement price. It is considered that a portion of the unfulfilled demand is lost and the other portion is backordered to the next period. In addition, all of the backordered demand at the end of the planning horizon is lost. A risk-averse decision maker is taken into consideration by whom at least a minimum portion of the arisen demand of each product should be fulfilled. In addition, it is considered that at least a minimum percentage of the fulfilled demand of each product should be satisfied exactly by the related product and cannot be fulfilled by the substitute products. The decision process is depicted in Figure 1.

\subsection{Proposed robust optimization framework}

Robust optimization method, proposed by Mulvey et al. [30], extends the traditional stochastic programming models by adding a variability measure to the expected 


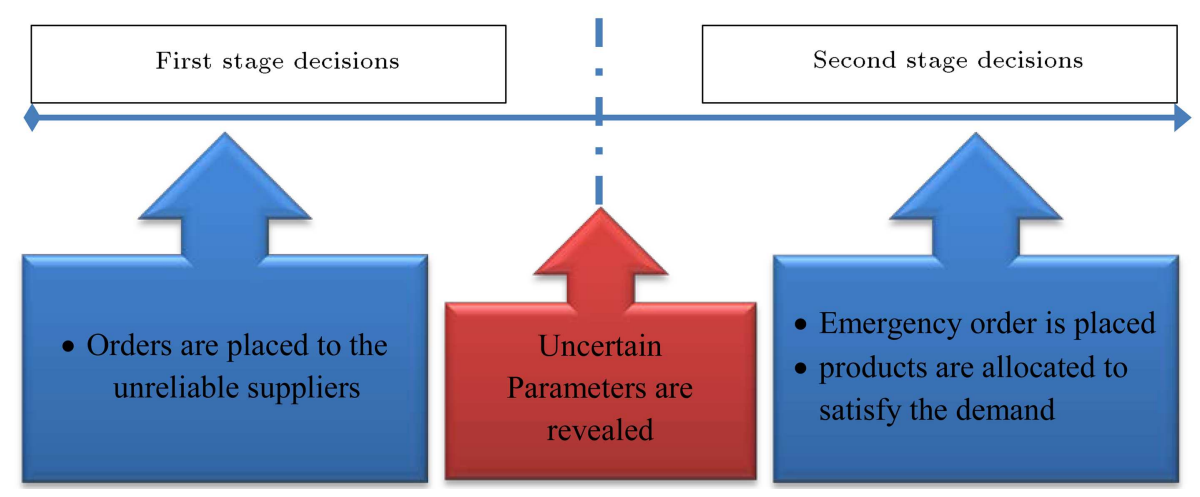

Figure 1. Decision process of the model.

profit measure. Such models will be robust optimal if they remain near optimal for the arisen scenarios. Such property is called solution robustness. In other words, solution robustness tends to decrease the variability of the stochastic model for $s \in S$. In addition, the above model will be robust with respect to feasibility if for all of the scenarios an almost feasible solution is obtained. Such property is called model robustness. The robust optimization framework, proposed by Mulvey [30], makes a tradeoff between solution robustness and model robustness. The robust optimization counterpart of a two-stage stochastic problem can be formulated as follows:

$$
\begin{aligned}
\max a^{T} x & +\sum_{s \in S} p^{s} \times b^{s T} \times y^{s}-\lambda \times \sigma\left(y^{1}, \ldots, y^{s}\right) \\
& -\omega \times \rho\left(\delta^{1}, \ldots, \delta^{S}\right)
\end{aligned}
$$

subject to:

$$
\begin{aligned}
& A x=c, \\
& B^{s} x+C^{s} \times y^{s}+\delta^{s}=d^{s}, \quad s \in S, \\
& x, y^{s} \geq 0, \quad s \in S,
\end{aligned}
$$

where $\sum_{s \in S} p^{s} \times b^{s T} \times y^{s}+\lambda \times \sigma\left(y^{1}, \ldots, y^{s}\right)$ indicates solution robustness of the objective function; $\lambda$ is a goal programing coefficient, which controls relative importance of variability measure for the decision maker; $\sigma\left(y^{1}, \ldots, y^{s}\right)$ is the variability measure of the recourse cost; $\rho\left(\delta^{1}, \ldots, \delta^{s}\right)$ is a penalty function, which decreases the violation of uncertain constraint; and $\omega$ is the weight of the goal programing model. In the next section, we present the proposed variability measures used for the proposed model.

\subsubsection{Variability measures for robust models}

In the classical robust optimization approach, mean variance measure of Markowitz [31] has been widely implemented to provide a tradeoff between variability and the expected profit. Due to the current limitations on quadratic solvers, it is not possible to solve large-size quadratic problems. Accordingly, several researchers have utilized linear variability measures instead of the quadratic variability measures [32]. Symmetric variability measures consider both upper and lower variations from a reference value, while several researchers have considered non-symmetric variability measures, which penalize only upper or lower variations from a reference target. For instance, List et al. [33] considered an upper partial moment of order 1 for fleet planning with uncertainty. Takriti and Ahmed [34] considered an upper partial moment of order 2 in a two-stage stochastic model. Having presented the most important variability measures, in the following section, the variability measures used in the proposed model are presented.

\subsubsection{Proposed variability measures}

It should be noted that for the problem proposed in this study, using a symmetric variability measure can generate inefficient solutions, which are not acceptable by a sagacious decision maker. The variability measures are determined based on lower partial variability measures as follows.

Partial Lower Deviation from Mean (PLDM)

A large portion of decision makers prefer lower profit partial variability, in which it is used to measure variability of the model for the cases with lower profit than the average profit. Partial Lower Deviation from Mean (PLDM) calculates the expected lower partial deviation from the mean by the following equation:

$$
\begin{aligned}
\sigma\left(y^{1}, \ldots, y^{s}\right)= & \sum_{s \in S} p^{s} \times \max \left(0, \sum_{s^{\prime} \in S} p^{s^{\prime}} \times\left(a^{T} x\right.\right. \\
& \left.\left.+b^{s^{\prime} T} \times y^{s^{\prime}}\right)-\left(a^{T} x+b^{s T} \times y^{s}\right)\right) .
\end{aligned}
$$

Partial Lower Deviation from Target (PLDT)

In addition to the PLDM variability measure, which considers the profit mean as the reference, Partial Lower Deviation from Target (PLDT) considers a 
predefined reference value instead of the mean profit. PLDT is defined by the following equation:

$$
\begin{aligned}
\sigma\left(y^{1}, \ldots, y^{s}\right)= & \sum_{s \in S} p^{s} \times \max \left(0, R^{*}-\left(a^{T} x\right.\right. \\
& \left.\left.+b^{s T} \times y^{s}\right)\right)
\end{aligned}
$$

where $R^{*}$ is the target of the objective function.

\subsection{The robust model}

The proposed two-stage model based on the assumptions presented in Section 3.2 is provided in the following:

\section{Indices}

$i, j \quad$ Item/product index $(i=1,2, \ldots, I$, $j=1,2, \ldots, J$ and $I=J)$

$t \quad$ Period index $(t=1,2, \ldots, T)$

$s, s^{\prime} \quad$ Scenario index

$k \quad$ Unreliable supplier index

\section{Parameters}

$S \quad$ Scenario set

$u_{i} \quad$ Set of suppliers that can supply item $i$

$I F_{i, 1} \quad$ The inventory level of item $i$ in the beginning of the planning horizon

$P U_{i, k, t} \quad$ Unit price of item $i$ from unreliable supplier $k$ in period $t$

$P E_{i, t} \quad$ Unit price of emergency supplier for item $i$ in period $t$

$R C_{i, t} \quad$ Unit reservation cost of item $i$ in period $t$

$D_{i, t} \quad$ Demand of item $i$ in period $t$

$P B_{i} \quad$ Unit backorder charge of item $i$

$P L_{i} \quad$ Unit lost sale charge of item $i$

$B V_{i} \quad$ Book value of item $i$ at the end of planning horizon

$I N_{i} \quad$ The income which is gained by unit demand satisfaction of item $i$

$\gamma_{i, j} \quad$ The quantity of item $j$, which is required to satisfy a unit of demand of item $i\left(\gamma_{i, i}=1\right)$

$Y_{i, k, t, s} \quad$ Yield of item $i$ from supplier $k$ in period $t$ for scenario $s$

$P R S_{s} \quad$ Probability of scenario $s$

$H_{i} \quad$ Holding cost of item $i$ for a period

A The percentage of unfulfilled demand that is backordered

$B \quad$ The percentage of demand which should be fulfilled and not backordered or lost $\theta$

The minimum percentage of demand that should be satisfied exactly by the related product

\section{Variables}

$I_{i, t, s} \quad$ Inventory level of item $i$ in the end of period $t$ for scenario $s$

$B_{i, t, s} \quad$ Backordered demand of item $i$ in period $t$ for scenario $s$

$L_{i, t, s} \quad$ Lost sale of item $i$ in period $t$ for scenario $s$

$F D_{i, t, s} \quad$ Fulfilled demand of item $i$ in period $t$ for scenario $s$

$O U_{i, k, t} \quad$ Order quantity to the unreliable supplier $k$ for product $i$ in period $t$

$O E_{i, t, s} \quad$ Order quantity to the emergency supplier for product $i$ in period $t$ with scenario $s$

$C_{i, j, t, s} \quad$ The consumed quantity of item $j$ to satisfy demand of product $i$ in period $t$ with scenario $s$

$R_{i, t} \quad$ Reserved quantity of item $i$ in period $t$

$\xi_{s} \quad$ The auxiliary variable which indicates the value of the second-stage objective function

$\varphi_{s} \quad$ The variability of each scenario from the reference

\section{Robust model}

The proposed robust model can be introduced as follow:

$$
\begin{aligned}
\max & \left\{-\sum_{t=1}^{T} \sum_{i=1}^{I} \sum_{k \in u_{i}} P U_{i, k, t} \times O U_{i, k, t}\right. \\
& -\sum_{t=1}^{T} \sum_{i=1}^{I} R C_{i, t} \times R_{i, t}+\sum_{s \in S} P R S_{s} \times \xi_{s} \\
& \left.-\lambda \sum_{s \in S} P R S_{s} \times \varphi_{s}^{P L D M}\right\},(P L D M), \\
\max \{ & -\sum_{t=1}^{T} \sum_{i=1}^{I} \sum_{k \in u_{i}} P U_{i, k, t} \times O U_{i, k, t} \\
& -\sum_{t=1}^{T} \sum_{i=1}^{I} R C_{i, t} \times R_{i, t} \\
& +\sum_{s \in S} P R S_{s} \times \xi_{s} \\
& \left.-\lambda \sum_{s \in S} P R S_{s} \times \varphi^{P L D T}\right\},(P L D T),
\end{aligned}
$$




$$
\begin{aligned}
\xi_{s}= & \sum_{i=1}^{I} \sum_{t=1}^{T} I N_{i} \times F D_{i, t, s}-\sum_{i=1}^{I} \sum_{t=1}^{T} P E_{i, t} \\
& \times O E_{i, t, s}-\sum_{i=1}^{I} \sum_{t=1}^{T} P B_{i} \times B_{i, t, s}-\sum_{i=1}^{I} \sum_{t=1}^{T} P L_{i} \\
& \times L_{i, t, s}-\sum_{i=1}^{I} P L_{i} \times B_{i, T, s}-\sum_{i=1}^{I} \sum_{t=1}^{T} H_{i} \times I_{i, t, s} \\
& +\sum_{i=1}^{I} B V_{i} \times I_{i, T, s}, \quad \forall s
\end{aligned}
$$$$
\left\{\begin{array}{c}
I F_{i, 1}+\sum_{k \in u_{i}} Y_{i, k, 1, s} \times O U_{i, k, 1}+O E_{i, 1, s} \\
=I_{i, 1, s}-B_{i, 1, s}+\sum_{j=1}^{J} C_{i, j, 1, s}, \quad \forall i, s \\
I_{i, t-1, s}-B_{i, t-1, s}+\sum_{k \in u_{i}} \times O U_{i, k, t} \\
+O E_{i, t, s}=I_{i, t, s}-B_{i, t, s}+\sum_{j=1}^{J} C_{i, j, t, s}, \\
\forall i, s, t=2, \ldots, T
\end{array}\right.
$$$$
F D_{i, t, s}+B_{i, t, s}+L_{i, t, s}=D_{i, t}, \quad \forall i, t, s,
$$$$
\sum_{j=1}^{J} \gamma_{i, j} \times C_{i, j, t, s}=F D_{i, t, s}, \quad \forall i, t, s
$$$$
(1-\alpha) \times B_{i, t, s}=\alpha \times L_{i, t, s}, \quad \forall i, t, s,
$$$$
\sum_{t=1}^{T} F D_{i, t, s} \geq \beta \times \sum_{t=1}^{T} D_{i, t, s}, \quad \forall i, t, s,
$$$$
C_{i, i, t, s} \geq \theta \times F D_{i, t, s}, \quad \forall i, t, s,
$$$$
O E_{i, t, s} \leq R_{i, t}, \quad \forall i, t, s
$$$$
\varphi_{s}^{P L D M} \geq \sum_{s^{\prime} \in S}\left(P R S_{s^{\prime}} \times\left(-\sum_{t=1}^{T} \sum_{i=1}^{I} \sum_{k \in u_{i}} P U_{i, k, t}\right.\right.
$$$$
\left.\left.\times O U_{i, k, t}-\sum_{t=1}^{T} \sum_{i=1}^{I} R C_{i, t} \times R_{i, t}+\xi_{s^{\prime}}\right)\right)
$$$$
-\left(-\sum_{t=1}^{T} \sum_{i=1}^{I} \sum_{k \in u_{i}} P U_{i, k, t} \times O U_{i, k, t}\right.
$$$$
\left.-\sum_{t=1}^{T} \sum_{i=1}^{I} R C_{i, t} \times R_{i, t}+\xi_{s}\right)
$$

$\forall s(P L D M)$,

$$
\begin{aligned}
& \varphi_{s}^{P L D T} \geq R^{*}-\left(-\sum_{t=1}^{T} \sum_{i=1}^{I} \sum_{k \in u_{i}} P U_{i, k, t} \times O U_{i, k, t}\right. \\
&\left.-\sum_{t=1}^{T} \sum_{i=1}^{I} R C_{i, t} \times R_{i, t}+\xi_{s}\right), \\
& \forall s(P L D T) I_{i, t, s}, B_{i, t, s}, L_{i, t, s}, F D_{i, t, s}, \\
& O U_{i, u, t}, O E_{i, t, s}, C_{i, j, t, s}, R_{i, t}, \varphi_{s}^{P L D M}, \\
& \varphi_{s}^{P L D T} \geq 0 .
\end{aligned}
$$

Eq. (1) is the objective function of the robust model, which is represented for both PLDM and PLDT variability measures. Eq. (2) shows the second-stage objective function. Eq. (3) represents the inventory balance for the planning horizon. Eq. (4) determines that the demand is fulfilled, backordered, or lost. Eq. (5) indicates that the fulfilled demand is fulfilled by exactly the related product or the substitute products. Eq. (6) indicates that a portion of the unfulfilled demand is backordered and the other portion is lost. Eq. (7) indicates that at least a portion of demand should be fulfilled and not backordered or lost. Eq. (8) indicates that at least a portion of the fulfilled demand should be satisfied exactly by the related product. Eq. (9) guaranties that the emergency orders do not exceed the reserved quantity. Eq. (10) determines the variability of the robust model. Eq. (11) indicates the non-negativity of variables.

\section{Case study}

In this section, we describe the results of the proposed model, which is implemented in an actual case. The models are coded in GAMS and solved with CPLEX 11 solver in a computer with $2.26 \mathrm{GHz}$ core 2 duo $\mathrm{CPU}$ and 3 GB RAM.

\subsection{Outline of the case}

The proposed robust optimization model is implemented in a distributor of pharmaceutical products located in Tehran, Iran, which distributes a variety of finished dosage drugs in the forms of tablets, capsules, syrup, and injection with different clinical therapeutic categories of antibiotics and non-antibiotic. Cephalosporin antibiotics, including Cefalexin and Cefixime, in different dosages and product forms are investigated in this study. The associated distributor receives the products mainly from several pharmaceutical companies based on their previous relationships or long-term contracts while emergency orders could be placed on other manufacturers or importers that can perfectly supply the related product from their available stock. The substitutability for a pharmaceutical 
case arises according to the allowed interchangeable use of drugs with similar clinical effects or the use of a similar drug with a different dosage form. The investigated distributor is concerned with 8 cephalosporin antibiotic products procured by 6 pharmaceutical companies (which are considered as the unreliable suppliers). The products under investigation are produced in the forms of tablets, capsules, and suspension.

\subsection{Results}

In Table 1, the numbers of engaged constraints and variables and the results of both two-stage and robust models are presented. As can be observed, the numbers of variables and constraints for both ordinary two-stage and robust models remain relatively close, indicating that similar solvers can be used for both the twostage and the robust models. In the rest of this study, the emergency ordering expenses include the reservation costs and the procurement charges of the emergency supplier. It is clear that by adding the variability measure, a lower net profit is obtained by the robust models, which arises because of the lower demand fulfillment income and higher reliance on the emergency supplier. In addition, the target value is determined by multiplication of a coefficient in the objective function of the pure two-stage model. In the following section, we first present the effect of emergency ordering and product substitutability and then present a detailed survey of the robust models.

\subsubsection{Effect of emergency ordering and product substitutability}

The emergency orders are directly affected by the price of the emergency orders while the portion of demand which is fulfilled by the substitute product can be controlled by the values of $\theta$. In the following sections, the values of emergency ordering and product substitutability are presented for $\lambda=0$.
Value of emergency ordering

Although considering the emergency ordering assumption increases the complexities of the mathematical model, it provides higher flexibilities for the decision maker, which leads to higher net profit. It is obvious that when the price of the emergency supplier increases, a lower quantity is ordered to the emergency supplier and after a threshold price for the emergency supplier, the model almost neglects the availability of emergency supplier because of its high price. To depict the behavior of the model for different emergency supplier prices, Emergency Price Coefficient (EPC) is utilized, which is multiplied in the original data to produce new emergency prices. In order to depict the effect of the new emergency prices, a criterion called Emergency Supplier Utilization (ESU) is formed, which determines the average ratio of orders to the emergency supplier and is calculated by the following equation:

$$
\begin{aligned}
E S U= & \sum_{i=1}^{I} \sum_{t=1}^{T} \sum_{s \in S} O E_{i, t, s} /\left(\sum_{i=1}^{I} \sum_{t=1}^{T} \sum_{s \in S} O E_{i, t, s}\right. \\
& \left.+\sum_{t=1}^{T} \sum_{i=1}^{I} \sum_{k \in u_{i}} O U_{i, k, t}\right) .
\end{aligned}
$$

Besides ESU criterion, the value of objective function shows the value that is added to the model for different values of EPC. The difference between the objective function for a high value of EPC and the actual data depicts the value of emergency ordering option. Figure 2 depicts the behavior of the model for different values of EPC.

It can be inferred from Figure 2 that as the price of the emergency source increases, a lower net profit is attained. In addition, it can be inferred that after a threshold value for EPC, the model is almost transformed into a model with only cheap unreliable

\begin{tabular}{|c|c|c|c|c|c|c|c|c|}
\hline & & $\begin{array}{c}\text { No. of } \\
\text { variables }\end{array}$ & $\begin{array}{c}\text { No. of } \\
\text { constraints }\end{array}$ & $\begin{array}{c}\text { Net } \\
\text { profit }\end{array}$ & $\begin{array}{c}\text { Overall } \\
\text { ordering } \\
\text { cost } \\
\end{array}$ & $\begin{array}{c}\text { Backordered/ } \\
\text { lost sale } \\
\text { cost }\end{array}$ & $\begin{array}{c}\text { Inventory } \\
\text { cost }\end{array}$ & $\begin{array}{c}\text { Demand } \\
\text { fulfillment } \\
\text { income } \\
\end{array}$ \\
\hline $\begin{array}{c}\text { Two-stage } \\
\text { model }(\lambda=0)\end{array}$ & & 303392 & 163296 & 16715.04 & 56145.83 & 6327.35 & 635.57 & 79823.79 \\
\hline \multirow{3}{*}{$\begin{array}{l}\text { Robust model } \\
\qquad(\lambda=0.1)\end{array}$} & PLDM & 304121 & 164025 & 13854.77 & 57052.79 & 6684.15 & 623.86 & 78215.57 \\
\hline & $\operatorname{PLDT}\left(R^{*}=90 \%\right)$ & 304121 & 164025 & 10592.79 & 57984.51 & 6652.65 & 639.61 & 75869.56 \\
\hline & $\operatorname{PLDT}\left(R^{*}=110 \%\right)$ & 304121 & 164025 & 11676.08 & 58560.55 & 6332.94 & 581.67 & 77151.24 \\
\hline \multirow{3}{*}{$\begin{array}{l}\text { Robust model } \\
\qquad(\lambda=0.6)\end{array}$} & PLDM & 304121 & 164025 & 4493.52 & 58984.67 & 8037.1 & 584.16 & 72099.45 \\
\hline & $\operatorname{PLDT}\left(R^{*}=90 \%\right)$ & 304121 & 164025 & 5486.37 & 58395.85 & 6882.48 & 685.37 & 71450.07 \\
\hline & $\operatorname{PLDT}\left(R^{*}=110 \%\right)$ & 304121 & 164025 & 6697.06 & 58683.88 & 6512.21 & 611.01 & 72504.16 \\
\hline
\end{tabular}

Table 1. Summary of the results of the robust model. 


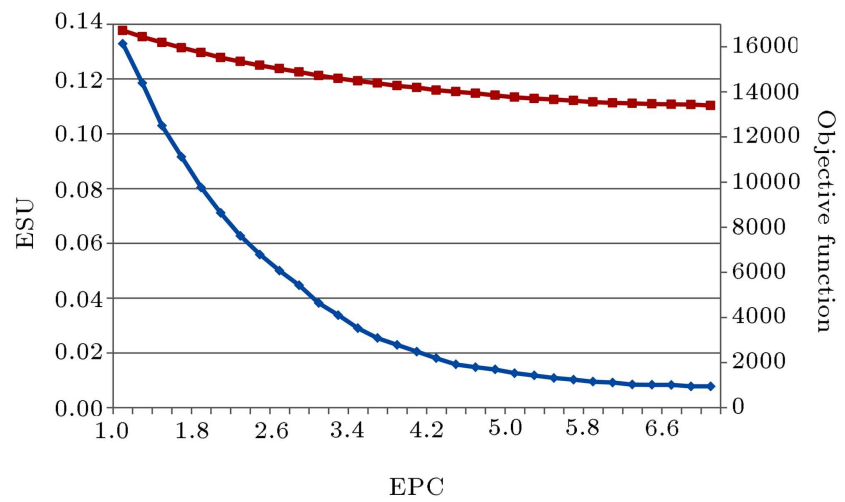

Figure 2. Effect of emergency supplier price on the model.

suppliers. Accordingly, the Value of Emergency Sourcing (VES) can be calculated by the difference between the profit gained by the actual case and the one with high value of EPC, which is calculated as follows:

$$
V E S=\text { Profit }_{E P C=1}-\text { Profit }_{E P C=7}=3321.18 .
$$

In addition, the portion of the total profit which is attained by emergency ordering is calculated in the following equation:

$$
V E S / \text { Profit }_{E P C=1}=3321.18 / 16715.04=19.84 \% .
$$

Accordingly, $19.84 \%$ of the profit is attained by flexibilities which are provided by emergency ordering option.

\section{Value of product substitutability}

Besides emergency ordering option, this paper presents product substitutability, which can provide higher flexibilities for the decision maker. It is clear that $\theta=1$ indicates a model which fully prohibits product substitutability while $\theta=0$ fully allows for product substitutability. The behavior of the profit function for different values of $\theta$ is depicted in Figure 3 .

The maximum Value of Product Substitutability (VPS) is computed as follows:

$$
V P S=\text { Profit }_{\theta=0}-\text { Profit }_{\theta=1}=864.43 .
$$

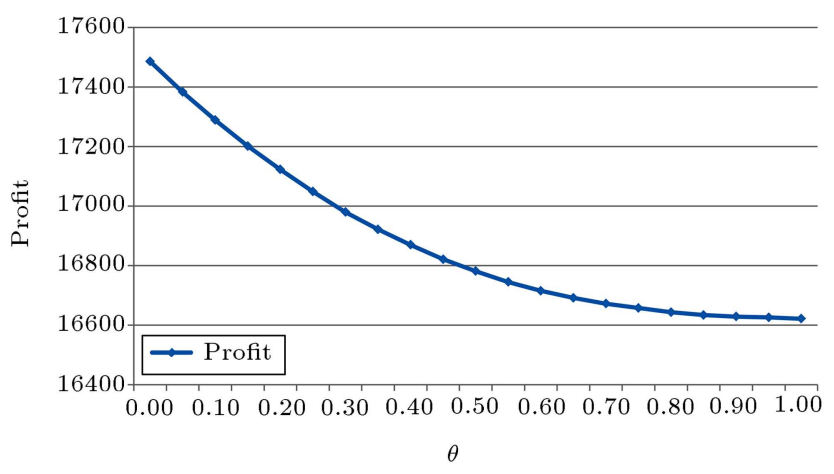

Figure 3. Effect of product substitutability on the net profit.

Accordingly, the maximum percentage of the profit which can be attained by product substitutability is computed as follows:

$$
V P S / \text { Profit }_{\theta=0}=864.43 / 16715.04=5.17 \% \text {. }
$$

\subsubsection{Effect of model robustness \\ PLDM model}

The results of the robust model with the PLDM variability measure are presented in Table 2 . The second to the seventh columns represent variability and parts of objective function including ordering cost, backordered/lost sale cost, inventory cost, and demand fulfillment income. As the value of $\lambda$ increases, the relative importance of the model robustness increases, which leads to the decrease in the PLDM variability. In addition, as the value of $\lambda$ increases, the ordering expenses to the unreliable suppliers decrease while the emergency ordering and the overall ordering expenses increase. This implies that as the value of $\lambda$ increases, the decision maker relies more on the expensive reliable supplier, which leads to the increase in the overall ordering expenses. Also, as the value of $\lambda$ increases, the backordered/lost sale expenses increase, too, which indicates that in order to decrease the variability, the model tends to fulfill a lower portion of the demand, which leads to the increase in backordered/lost sale expenses. The result indicates that as $\lambda$ increases,

Table 2. Summary of the results of the robust model with PLDM variability measure.

\begin{tabular}{lcccccc}
\hline $\boldsymbol{\lambda}$ & $\begin{array}{c}\text { Unreliable } \\
\text { supplier } \\
\text { ordering cost }\end{array}$ & $\begin{array}{c}\text { Expected } \\
\text { emergency } \\
\text { ordering cost }\end{array}$ & $\begin{array}{c}\text { Expected } \\
\text { backordered/ } \\
\text { lost sale cost }\end{array}$ & $\begin{array}{c}\text { Expected } \\
\text { inventory } \\
\text { cost }\end{array}$ & $\begin{array}{c}\text { Expected } \\
\text { demand } \\
\text { fulfillment } \\
\text { income }\end{array}$ & $\begin{array}{c}\text { Expected } \\
\text { variability } \\
\text { measure }\end{array}$ \\
\hline 0.1 & 47230.19 & 9822.6 & 6684.15 & 623.86 & 78215.57 & 2084.07 \\
0.2 & 46767.07 & 11037.67 & 7021.99 & 612.74 & 76707.58 & 1780.45 \\
0.3 & 46314.19 & 11973.88 & 7318.85 & 601.32 & 75240.64 & 1583.20 \\
0.4 & 46041.27 & 12625.27 & 7591 & 595.3 & 73973.39 & 1454.95 \\
0.5 & 45878.53 & 13073.6 & 7814.92 & 588.63 & 72936.72 & 1353.70 \\
0.6 & 45612.13 & 13372.54 & 8037.1 & 584.16 & 72099.45 & 1287.45 \\
\hline
\end{tabular}




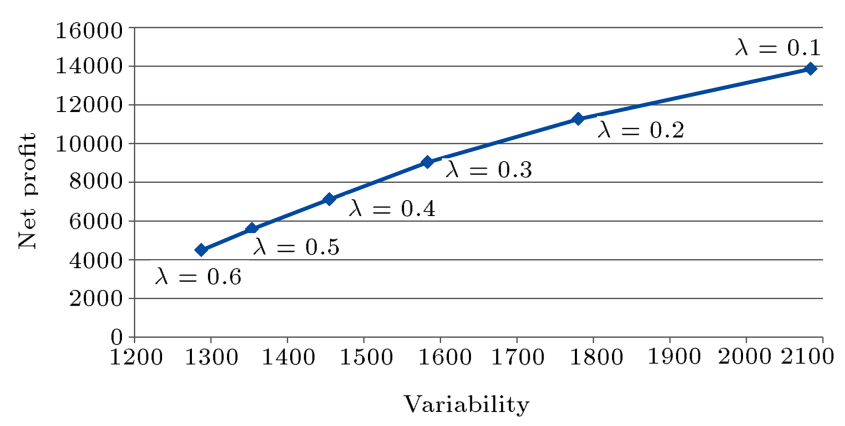

Figure 4. The mutual effect of variability and net profit.

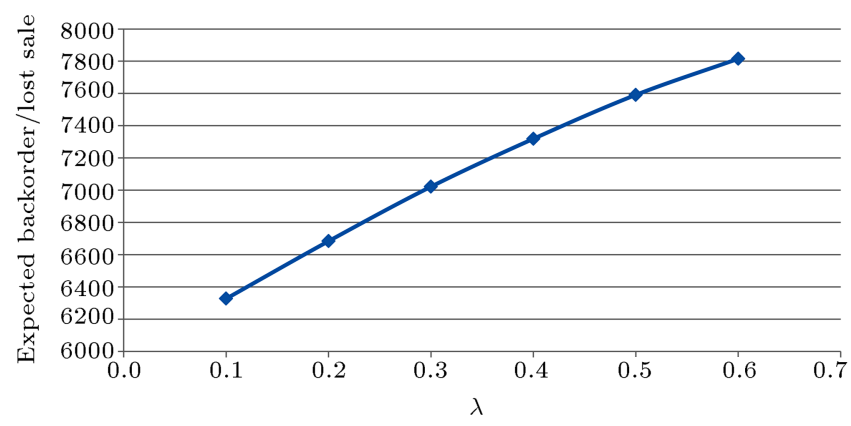

Figure 5. Risk-aversion effect on the selection of $\lambda$.

the overall order quantity, which includes both order quantities to the reliable and unreliable suppliers, decreases. In addition, due to the short length of the planning periods, the inventory holding costs constitute only a small portion of the overall expenses. Table 2 shows the results for PLDM variability measure, which verifies the above discussions.

It should be noted that while a decision maker wishes to have a robust model, he/she should choose a value for $\lambda$ which reflects his/her attitude toward profitability or the required service level, too. Figure 4 illustrates the relation between variability and the overall net profit. It can be inferred that as the desire of the decision maker to obtain a more robust model increases, a lower net profit is attained.

On the other hand, it is obvious that lower backordered/lost sales expenses correspond with a higher service level. Figure 5 shows that as $\lambda$ increases, a higher expected backordered/lost sale is determined by the model. Accordingly, by increase in the value of $\lambda$, a lower service level is obtained. In summary, it could be concluded that while the decision maker desires a robust solution regardless of profit or service level, a high value of $\lambda$ should be selected. Also, if the decision maker desires to achieve a solution which corresponds with higher service level and net profit, a low value of $\lambda$ should be implemented in the model.

\section{PLDT variability measure}

In this study, the target value for PLDT model represents a yardstick in which the decision maker desires to minimize variability of solutions, which has a net profit lower than it. In order to investigate the behavior of the model against perturbations in the values of $R^{*}$ and $\lambda$, a range of values are generated for both $R^{*}$ and $\lambda$. The value of $\lambda$ depicts the importance of solution robustness for the decision maker while $R^{*}$ depicts the profit goal. Table 3 depicts a summary of the results of the model for a set of values of $\lambda$ and $R^{*}$.

As it is expected, for a given value of $R^{*}$, as $\lambda$ increases, the variability measure decreases. Thus, by the increase in $\lambda$, better control is provided on the variability of solutions similar to PLDM model. In addition, as the value of $\lambda$ increases, unreliable supplier ordering expenses decrease while the expected emergency ordering and the overall ordering expenses increase. The increase in the expected emergency supplier expenses indicates that as the value of $\lambda$ increases, the model takes the advantage of reliability of the reliable emergency supplier more. However, higher utilization of reliable suppliers besides lower utilization of unreliable suppliers results in the decrease of the inventory holding costs. In addition, the increase in $\lambda$ results in higher expected backordered/lost sale expenses. The increase in both the expected backordered/lost sale and the overall ordering expenses indicates that as $\lambda$ increases, a lower overall quantity is ordered to both reliable and unreliable suppliers. Finally, it should be noted that as the value of $\lambda$ increases, both demand fulfillment income and the net profit decrease.

On the other hand, for a fixed value of $\lambda$, as $R^{*}$ increases, the ordering expenses to the unreliable suppliers decrease while the emergency ordering and the overall ordering expenses increase. However, by the increase of $R^{*}$, the backordered/lost sale and the inventory holding expenses decrease while the net profit of the model increases. In addition, the variability of the model increases by the increase of $R^{*}$. Figure 6 illustrates the tradeoff between the expected profit and PLDT variability for different values of $R^{*}$. It can be inferred that by the increase of the target value $\left(R^{*}\right)$, a higher variability is determined by the model. Accordingly, if the decision maker desires a more robust solution with lower variability, a low value should be selected for $R^{*}$. Figure 7 depicts the tradeoff between the overall ordering expenses and the expected backordered/lost sale expenses for different values of $R^{*}$, which indicates that for a fixed value of $\lambda$, as the expected overall ordering charge increases, lower expected backordered/lost sale expenses are determined by the model. In addition, for a given $\lambda$, as $R^{*}$ increases, lower backordered/lost sale expense is obtained. In summary, it could be concluded that $\lambda$ and $R^{*}$ should be selected in a way that reflects the decision maker's attitudes toward, for instance, risk averseness, attained net profit, variability, etc. Accordingly, if the decision maker desires to minimize variability, he/she should 
Table 3. Summary of the results of the robust model with PLDT variability measure.

\begin{tabular}{|c|c|c|c|c|c|c|c|}
\hline $\boldsymbol{R}^{*}$ & $\lambda$ & $\begin{array}{c}\text { Unreliable } \\
\text { supplier } \\
\text { ordering cost }\end{array}$ & $\begin{array}{c}\text { Expected } \\
\text { emergency } \\
\text { ordering cost }\end{array}$ & $\begin{array}{c}\text { Expected } \\
\text { backordered/ } \\
\text { lost sale cost }\end{array}$ & $\begin{array}{c}\text { Expected } \\
\text { inventory } \\
\text { cost }\end{array}$ & $\begin{array}{c}\text { Expected } \\
\text { demand } \\
\text { fulfillment income }\end{array}$ & $\begin{array}{c}\text { Expected } \\
\text { variability } \\
\text { measure }\end{array}$ \\
\hline \multirow{6}{*}{$R^{*}=90 \%$} & 0.1 & 47282.86 & 10701.65 & 6652.65 & 639.61 & 75869.56 & 1791.209 \\
\hline & 0.2 & 46488.38 & 11601.93 & 6726.01 & 650.29 & 74775.25 & 1699.276 \\
\hline & 0.3 & 45948.75 & 12241.25 & 6812.62 & 661.7 & 73773.46 & 1613.07 \\
\hline & 0.4 & 45539.23 & 12739.81 & 6842.13 & 670.98 & 72891.32 & 1535.725 \\
\hline & 0.5 & 45194.31 & 13161.85 & 6863.5 & 679.16 & 72120.11 & 1471.586 \\
\hline & 0.6 & 45139.46 & 13256.39 & 6882.48 & 685.37 & 71450.07 & 1417.444 \\
\hline \multirow{6}{*}{$R^{*}=95 \%$} & 0.1 & 46836.38 & 11350.2 & 6610.51 & 627.39 & 76342.04 & 1835.706 \\
\hline & 0.2 & 46130.81 & 12116.1 & 6679.24 & 638.67 & 75099.77 & 1745.663 \\
\hline & 0.3 & 45772.41 & 12548.26 & 6741.32 & 647.39 & 74078.32 & 1662.577 \\
\hline & 0.4 & 45419.05 & 12965.88 & 6778.44 & 652.95 & 73240.81 & 1591.644 \\
\hline & 0.5 & 45119.11 & 13299.55 & 6817.61 & 661.19 & 72500.87 & 1525.889 \\
\hline & 0.6 & 45039.31 & 13416.07 & 6829.14 & 669.27 & 71795.86 & 1469.074 \\
\hline \multirow{6}{*}{$R^{*}=100 \%$} & 0.1 & 46490.6 & 11831.87 & 6531.35 & 611.26 & 76681.9 & 1889.004 \\
\hline & 0.2 & 45985.65 & 12378.29 & 6602.52 & 623.02 & 75397.85 & 1796.38 \\
\hline & 0.3 & 45640.14 & 12770.18 & 6667.27 & 632.55 & 74407.91 & 1714.187 \\
\hline & 0.4 & 45262.41 & 13201.79 & 6707.13 & 639.2 & 73606.16 & 1647.864 \\
\hline & 0.5 & 45021.5 & 13486.45 & 6723.2 & 641.01 & 72777.28 & 1581.236 \\
\hline & 0.6 & 45002.99 & 13533.39 & 6743.58 & 643.55 & 72020.87 & 1513.53 \\
\hline \multirow{6}{*}{$R^{*}=105 \%$} & 0.1 & 46401.86 & 12064.34 & 6421.18 & 593.49 & 76881.38 & 1939.3 \\
\hline & 0.2 & 45980.76 & 12505.25 & 6482.1 & 599.46 & 75685.59 & 1852.333 \\
\hline & 0.3 & 45598.61 & 12918.32 & 6571.12 & 606.89 & 74665.11 & 1771.05 \\
\hline & 0.4 & 45244.31 & 13300.58 & 6602.79 & 611.87 & 73857.89 & 1702.329 \\
\hline & 0.5 & 45012.68 & 13566.68 & 6623.57 & 617.98 & 73027.46 & 1638.009 \\
\hline & 0.6 & 44997.57 & 13609.36 & 6638.71 & 621.63 & 72282.44 & 1577.871 \\
\hline \multirow{6}{*}{$R^{*}=110 \%$} & 0.1 & 46217.1 & 12343.45 & 6332.94 & 581.67 & 77151.24 & 2009.109 \\
\hline & 0.2 & 45653.34 & 12934.8 & 6377.34 & 587.57 & 75952.49 & 1921.425 \\
\hline & 0.3 & 45359.24 & 13268.54 & 6420.77 & 596 & 74923.7 & 1838.018 \\
\hline & 0.4 & 45243.34 & 13409.57 & 6442.15 & 602.95 & 74076.71 & 1770.172 \\
\hline & 0.5 & 44911.58 & 13759.19 & 6474.52 & 609.39 & 73327.82 & 1706.131 \\
\hline & 0.6 & 44850.65 & 13833.23 & 6512.21 & 611.01 & 72504.16 & 1638.066 \\
\hline
\end{tabular}

select a model with a low value of $R^{*}$ and high value of $\lambda$ while for a service-sensitive decision maker, a high value of $R^{*}$ and low value of $\lambda$ should be implemented.

The presented framework is valid in depicting the behavior of the model for different values of $R^{*}$ and $\lambda$ for other cases (e.g., data of other companies) and only the scale of the values might be changed. In such cases, it is possible to run the model for several values of $R^{*}$ and $\lambda$ initially and by observing the behavior of the model ingredients, it is possible to narrow the values of $\lambda$ and $R^{*}$ based on managers' decision. This means that in using other data, it is possible to use other ranges for $\lambda$ and $R^{*}$. However, in such cases, the behavior of the ingredients of the models remains the same.

\section{Comparison between PLDM and PLDT variability} measures

In this part, the behavior of the PLDM and PLDT models are compared in a way that directs the decision maker to select the proper model. As explained in the previous sections, in the PLDM model, the variability measure basically depends only on the values of $\lambda$ while in the PLDT model, the variability depends on both $R^{*}$ and $\lambda$. As it is expected, when the target value is not restricted to a predetermined value (PLDM model), as the value of $\lambda$ increases, a higher control on the variability is acquired while in the PLDT models, for a given value of $R^{*}$, as $\lambda$ increases, variability slightly decreases. In addition, for a given value of $\lambda$, increase in $R^{*}$ results in increase in PLDT variability. 


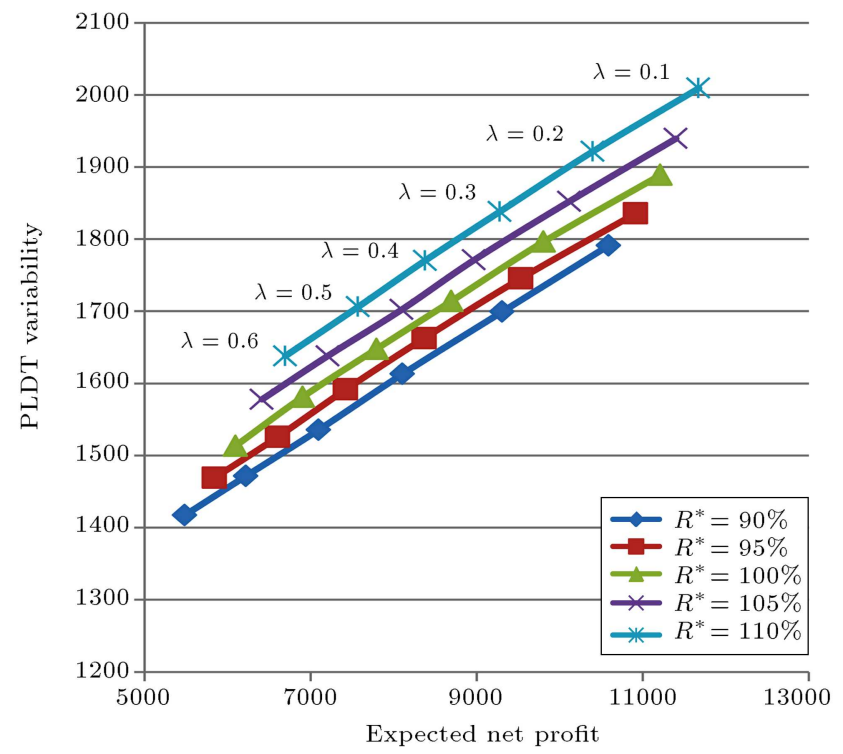

Figure 6. Mutual effect of the PLDT variability and the expected net profit.

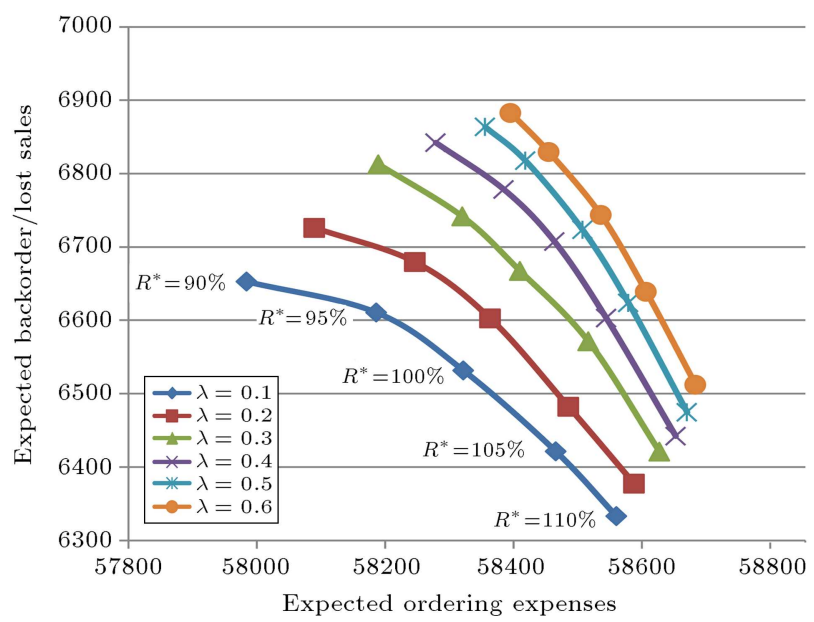

Figure 7. Mutual effect of expected backordered/lost sale and expected ordering expenses.

On the other hand, for a given value of $R^{*}$, as the value of $\lambda$ increases, the backordered/lost sale expenses increase slightly while for a given value of $\lambda$, as $R^{*}$ increases, a lower backordered/lost sale is obtained for the problem. In addition, for PLDM models, a higher backordered/lost sale is achieved. Also, as the value of $\lambda$ increases, the net profit of both PLDM and PLDT models decreases. However, the decrease rate for the PLDM model is higher. Figures 8-10 depict the variability, backordered/lost sales, and net profit of PLDM and PLDT models for different values of $R^{*}$ and $\lambda$.

In order to illustrate the fact that which model should be selected by certain practitioners, a comparison has been performed between PLDM and PLDT models. In a service-sensitive company that desires high customer satisfaction, the decision maker should

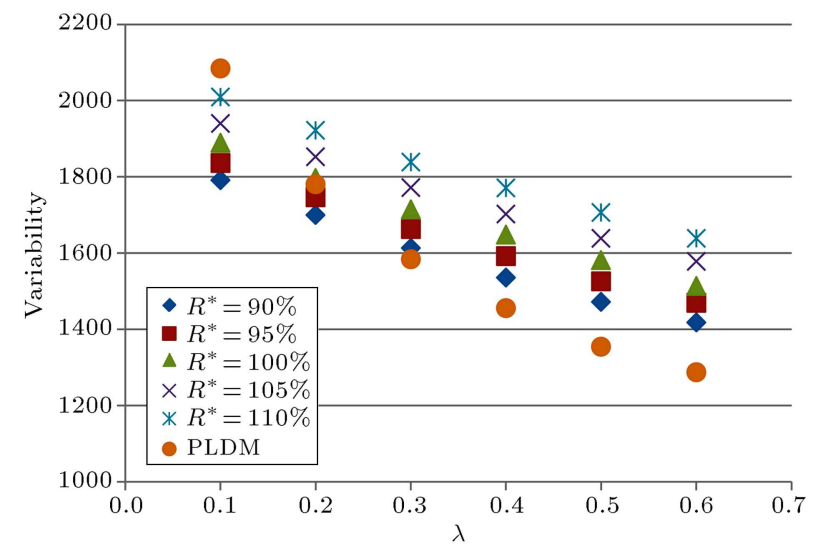

Figure 8. Effect of robust model parameters on variability.

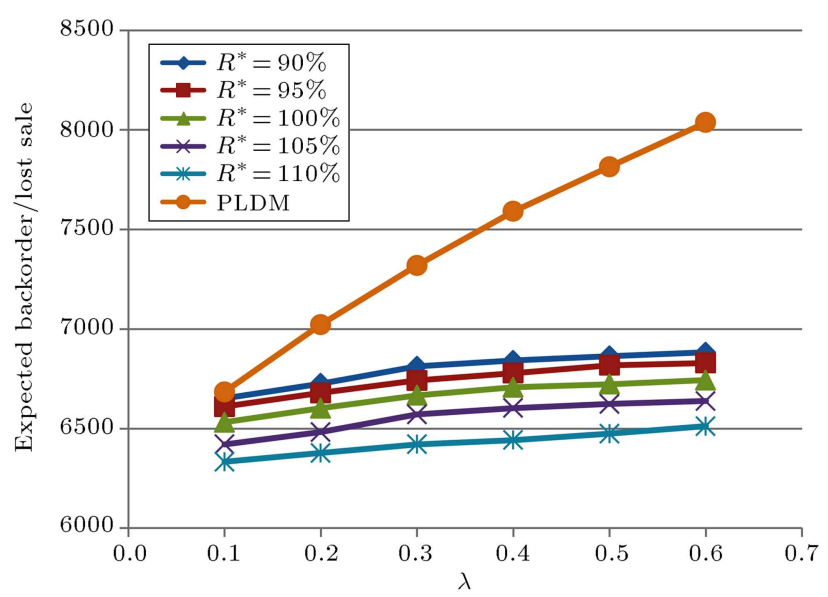

Figure 9. Effect of robust model parameters on the expected backordered/lost sale.

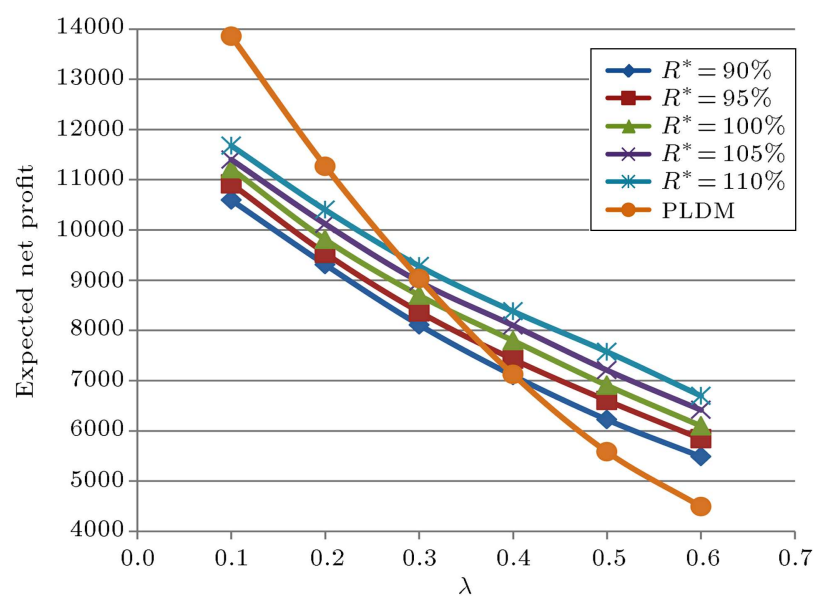

Figure 10. Effect of robust model parameters on the expected profit.

select a model which results in the lowest backordered/lost sale expenses. In the experiment, the PLDT model with a high value of $R^{*}$ and low value of $\lambda$ results in the least backordered/lost sale expenses. Although such model results in lower backorder charges, 
it results in relatively high variability, which is not desired for a decision maker who seeks for a robust model with low variability. On the other hand, if the decision maker desires the highest average net profit regardless of backordered/lost sale and variability, a PLDM model with a small value of $\lambda$ should be utilized and if the decision maker desires to achieve a robust solution with a relatively small variability, a PLDM model with a high value of $\lambda$ should be implemented. However, the PLDM model with a high value of $\lambda$ results in higher expected ordering and backordered/lost sales expenses, which indicates a low customer service level.

In the above discussion, the behavior of the model was studied based on real data. Managers and practitioners who are going to use the proposed model should precisely consider the fact that by using other data sets, the behavior of the model remains the same but the scale of the model might be changed. Accordingly, to determine the values of $\lambda$ and $R^{*}$ in other problems, it is proposed that, initially, the decision maker run several problems with a wide range of $\lambda$ and $R^{*}$ with different values to check the variation of the model ingredients and, in the next step, based on the achieved results, the practitioner select a narrow range of $\lambda$ and $R^{*}$ and precisely determine the final values of $\lambda$ and $R^{*}$.

\section{Conclusion}

This paper presents a two-stage stochastic model for an inventory problem with uncertainty. The orders can be placed on both expensive reliable supplier and cheap unreliable suppliers prone to yield uncertainty. The demand can be fulfilled directly by the related product or the substitute product. A robust optimization approach with PLDM and PLDT variability measures is proposed. Our experiment reveals the behavior of the model for each of the proposed variability measures and specifies the proper model based on attitude of decision maker against the desired backordered/lost sale, profitability, and solution robustness. It is clear that it is not possible to attain a model which concurrently maximizes the net profit and minimizes the variability and backordered/lost sale expenses. Accordingly, in any practical implementations, a tradeoff between the above measures should be achieved according to the desire of the decision maker. According to the results of this experiment, if the decision maker desires to maximize the net profit regardless of the backordered/lost sale or the variability of the model, a PLDM model with a small value of $\lambda$ should be selected. Also, if the decision maker desires to minimize the backordered/lost sales, a PLDT model with a high value of $R^{*}$ and low value of $\lambda$ should be selected while if the main desire is to minimize variability, a PLDM model with high value of $\lambda$ should be selected.

Although the proposed variability measures work based on the first order of deviation, which makes it possible for the model to be solved by LP solvers, it is possible to use second-order variability measures, which necessitates a quadratic solver. According to the current limitations on quadratic solvers, it is not possible to solve large-size problems by the current commercial quadratic solvers. In such cases, it is possible to use approximation methods, such as Sample Average Approximation, which deal with a smaller number of scenarios. Although the utilization of quadratic measures results in a more complicated model, it can provide better control on the variability of model.

In addition, although the above model is implemented for a pharmaceutical distributor, it can be implemented in a variety of other applications including grocery or semiconductor industries in which similar assumptions can be implemented. Finally, it should be noted that the majority of the inventory models with disruption in the literature are modeled in a way that is not easily extendable for similar assumptions while this paper presents a flexible mathematical model which can easily embody other assumptions. In addition, the proposed model excels the previous inventory disruption models by considering a variability measure which is not easily adaptable to the previous models. Accordingly, it is strongly recommended that the proposed method be considered before implementing other methods.

\section{Acknowledgement}

The authors would like to acknowledge the partial financial support of the Iran National Science Foundation under Grant No. 94813083.

\section{References}

1. Reitman, V. "Toyota motor shows its mettle after fire destroys parts plant", Wall Street Journal, 8, p. A-1 (1997).

2. Latour, A. "Trial by fire: A blaze in Albuquerque sets off major crisis for cell-phone giants", Wall Street Journal, 1(29) (2001).

3. Tomlin, B. and Wang, Y. "Operational strategies for managing supply chain disruption risk", The Handbook of Integrated Risk Management in Global Supply Chains, pp. 79-101 (2011).

4. Gibb, F. and Buchanan, S. "A framework for business continuity management", International Journal of Information Management, 26(2), pp. 128-141 (2006).

5. Qi, L. and Shen, Z.J.M. "A supply chain design model with unreliable supply", Naval Research Logistics (NRL), 54(8), pp. 829-844 (2007). 
6. Mukhopadhyay, S.K. and Ma, H. "Joint procurement and production decisions in remanufacturing under quality and demand uncertainty", International Journal of Production Economics, 120(1), pp. 5-17 (2009).

7. Agrawal, N. and Nahmias, S. "Rationalization of the supplier base in the presence of yield uncertainty", Production and Operations Management, 6(3), pp. 291-308 (1997).

8. Dada, M., Petruzzi, N.C. and Schwarz, L.B. "A newsvendor's procurement problem when suppliers are unreliable", Manufacturing \& Service Operations Management, 9(1), pp. 9-32 (2007).

9. Maddah, B., Salameh, M.K. and Karame, G.M. "Lot sizing with random yield and different qualities", Applied Mathematical Modelling, 33(4), pp. 1997-2009 (2009).

10. Li, X. and Zhang, X. "Emergency procurement based on supply disruptions: Single-sourcing or dualsourcing", Open Cybernetics \& Systemics Journal, 8, pp. 618-622 (2014).

11. Xiao, T. and Qi, X. "Price competition, cost and demand disruptions and coordination of a supply chain with one manufacturer and two competing retailers", Omega, 36(5), pp. 741-753 (2008).

12. Zeynep Sargut, F. and Qi, L. "Analysis of a two-party supply chain with random disruptions", Operations Research Letters, 40(2), pp. 114-122 (2012).

13. Tomlin, B. and Wang, Y. "On the value of mix flexibility and dual sourcing in unreliable newsvendor networks", Manufacturing \& Service Operations Management, 7(1), pp. 37-57 (2005).

14. Tomlin, B. "On the value of mitigation and contingency strategies for managing supply chain disruption risks", Management Science, 52(5), pp. 639-657 (2006).

15. Iakovou, E., Vlachos, D., Keramydas, C. and Tsiolias, D. "Evaluation of emergency sourcing risk mitigation strategies for a discrete part manufacturer", International Journal of Advanced Logistics, 4(1), pp. 37-46 (2015).

16. Keramydas, C., Tsiolias, D., Vlachos, D. and Iakovou, E. "A simulation methodology for evaluating emergency sourcing strategies of a discrete part manufacturer", International Journal of Data Analysis Techniques and Strategies, 7(2), pp. 141-155 (2005).

17. Chen, K. and Yang, L. "Random yield and coordination mechanisms of a supply chain with emergency backup sourcing", International Journal of Production Research, 52(16), pp. 4747-4767 (2014).

18. Mardan, E., Amalnik, M.S. and Rabbani, M. "An integrated emergency ordering and production planning optimization model with demand and yield uncertainty", International Journal of Production Re- search, (ahead-of-print), 53(20), pp. 6023-6039 (2015).

19. Zhang, Y., Long, J. and Shi, C. "A comprehensive contingency management framework for supply chain disruption risk management" International Journal of Automation and Logistics, 1(4), pp. 343-369 (2016).

20. Glud Johansen, S. and Thorstenson, A. "Emergency orders in the periodic-review inventory system with fixed ordering costs and compound Poisson demand", International Journal of Production Economics, 157, pp. 147-157 (2014).

21. Huang, S., Axsäter, S., Dou, Y. and Chen, J. "A realtime decision rule for an inventory system with committed service time and emergency orders", European Journal of Operational Research, 215(1), pp. 70-79 (2011).

22. Axsäter, S. "An improved decision rule for emergency replenishments", International Journal of Production Economics, 157, pp. 313-317 (2014).

23. Bassok, Y., Anupindi, R. and Akella, R. "Single-period multiproduct inventory models with substitution", $O p$ erations Research, 47(4), pp. 632-642 (1999).

24. Ganesh, M., Raghunathan, S. and Rajendran, C. "The value of information sharing in a multi-product, multilevel supply chain: Impact of product substitution, demand correlation, and partial information sharing", Decision Support Systems, 58, pp. 79-94 (2014).

25. Iravani, S.M., Kolfal, B. and Van Oyen, M.P. "Process flexibility and inventory flexibility via product substitution", Flexible Services and Manufacturing Journal, 26(3), pp. 320-343 (2012).

26. Huang, D., Zhou, H. and Zhao, Q.-H. "A competitive multiple-product newsboy problem with partial product substitution", Omega, 39(3), pp. 302-312 (2011).

27. Liu, W., Song, S. and Wu, C. "Impact of loss aversion on the newsvendor game with product substitution", International Journal of Production Economics, 141(1), pp. 352-359 (2013).

28. Goyal, M. and Netessine, S. "Volume flexibility, product flexibility, or both: The role of demand correlation and product substitution", Manufacturing \& Service Operations Management, 13(2), pp. 180-193 (2011).

29. Yano, C.A. and Lee, H.L. "Lot sizing with random yields: A review", Operations Research, 43(2), pp. 311-334 (1995).

30. Mulvey, J.M., Vanderbei, R.J. and Zenios, S.A. "Robust optimization of large-scale systems", Operations Research, 43(2), pp. 264-281 (1995).

31. Markowitz, H., Portfolio Selection: Efficient Diversification of Investments, New York 1959. 
32. Yu, C.-S. and Li, H.-L. "A robust optimization model for stochastic logistic problems", International Journal of Production Economics, 64(1), pp. 385-397 (2000).

33. List, G.F., et al., "Robust optimization for fleet planning under uncertainty", Transportation Research Part E: Logistics and Transportation Review, 39(3), pp. 209-227 (2003).

34. Takriti, S. and Ahmed, S. "On robust optimization of two-stage systems", Mathematical Programming, 99(1), pp. 109-126 (2004).

\section{Biographies}

Ehsan Mardan received his BSc degree in Industrial Engineering in 2008 from Amirkabir University of Technology, Tehran, Iran, and his MSc and PhD degree from the Department of Industrial Engineering, College of Engineering, University of Tehran, Iran. His research interests include supply chain management, inventory systems, and disruption.

Mohsen Sadegh Amalnick received his BSc degree in Electrical Engineering in 1980 from the State University of New York, USA, and his MSc degree in Industrial Engineering and Manufacturing Engineering in 1996 from Moscow State Technical University, Russia. He also received his $\mathrm{PhD}$ degree in Industrial Engineering and Manufacturing Engineering in 1999 from Russian Academy of Sciences, Russia. He is an
Associate Professor in the Department of Industrial Engineering, College of Engineering, University of Tehran, Iran. His research interests include information technology, knowledge management, automation systems, CAD/CAM and flexible production systems, and new product development.

Masoud Rabbani is a Professor of Industrial Engineering in the School of Industrial and Systems Engineering at University of Tehran. He has published more than 60 papers in international journals, such as European Journal of Operational Research, International Journal of Production Research, and International Journal of Production Economics, among others. His research interests include production-planning, design of inventory management systems, applied graph theory in industrial planning, and productivity management.

Fariborz Jolai received BS and MS degrees in Industrial Engineering from Amirkabir University of Technology, Tehran, Iran, in 1986 and 1989, respectively, and a $\mathrm{PhD}$ degree in Industrial Engineering from the Institute National Polytechnique de Grenoble, Grenoble, France, in 1998. He is currently Professor in the Industrial Engineering Department at Tehran University, Tehran, Iran. His research interests include scheduling, supply chain management, inventory control, and facility location. 